\title{
Novel Cellulose-Based Amorphous Solid Dispersions Enhance Quercetin Solution Concentrations In Vitro
}

\author{
Andrew D. Gilley ${ }^{\mathrm{a}}$, Hale Cigdem Arca ${ }^{\mathrm{b}}$, Brittany L.B. Nichols ${ }^{\mathrm{d}}$, Laura I. Mosquera-Giraldo ${ }^{\mathrm{e}}$, \\ Lynne S. Taylor ${ }^{\mathrm{e}}$, Kevin J. Edgar ${ }^{\mathrm{b}, \mathrm{c}}$, Andrew P. Neilson ${ }^{\mathrm{a}, *}$ \\ ${ }^{a}$ Department of Food Science and Technology, Virginia Tech, Blacksburg, VA 24060; \\ ${ }^{b}$ Macromolecules Innovation Institute, Virginia Tech; \\ ${ }^{\mathrm{c}}$ Department of Sustainable Biomaterials, Virginia Tech; \\ ${ }^{\mathrm{d}}$ Department of Chemistry, Virginia Tech; \\ ${ }^{\mathrm{e}}$ Department of Industrial and Physical Pharmacy, Purdue University, West Lafayette, IN 47907
}

*Corresponding author: Dr. Andrew P. Neilson, Dept. of Food Science and Technology, Virginia Tech, 1981 Kraft Dr., Blacksburg, VA 24060; E-mail: andrewn@vt.edu; Phone: (540) 231-8391.

\footnotetext{
Abbreviations: Amorphous solid dispersion (ASD), carboxycellulose acetate butyrate (CCAB), cellulose acetate suberate (CASub), epicatechin (EC), hydroxypropylmethyl cellulose acetate succinate (HPMCAS) polyvinylpyrrolidone (PVP)
} 


\section{ABSTRACT}

27 Quercetin (Q) is a bioactive flavonol with potential to benefit human health. However, Q 28 bioavailability is relatively low, due to its poor aqueous solubility and extensive phase-II 29 metabolism. Strategies to increase solution concentrations in the small intestinal lumen have the 30 potential to substantially increase Q bioavailability, and by extension, efficacy. We aimed to 31 achieve this by incorporating Q into amorphous solid dispersions (ASDs) with cellulose 32 derivatives. Q was dispersed in matrices of cellulose esters including 6-carboxycellulose acetate 33 butyrate (CCAB), hydroxypropylmethylcellulose acetate succinate (HPMCAS) and cellulose 34 acetate suberate (CASub) to afford ASDs that provided stability against crystallization, and $\mathrm{pH}-$ 35 triggered release. Blends of CASub and CCAB with the hydrophilic polyvinylpyrrolidone (PVP) 36 further enhanced dissolution. The ASD 10\% Q:20\% PVP:70\% CASub most significantly 37 enhanced $\mathrm{Q}$ solution concentration under intestinal $\mathrm{pH}$ conditions, increasing area under the 38 concentration/time curve (AUC) 18-fold compared to Q alone. This novel ASD method promises 39 to enhance $\mathrm{Q}$ bioavailability in vivo.

Keywords: Amorphous solid dispersion, cellulose, quercetin, dissolution, bioavailability, solubility enhancement

\section{Chemical compounds studied in this article}

Quercetin (PubChem CID: 5280343), hydroxypropylmethylcellulose acetate succinate 48 (PubChem CID: 121950), polyvinylpyrrolidone (PubChem CID: 6917) 


\section{INTRODUCTION}

Q (Fig. 1) is a dietary flavonol (a subclass of flavonoids) present at high levels in foods including apples, onions, and broccoli (Caridi et al., 2007; Price, Casuscelli, Colquhoun, \& Rhodes, 1998). Q intake has been associated with many potential health benefits, including reduced risk of cardiovascular disease (Russo, Spagnuolo, Tedesco, Bilotto, \& Russo, 2012), cancer (Firdous et al., 2014; Jaganathan, 2011; Zhou et al., 2010), and diabetes and obesity (Chiş, Baltaru, Maier, Mureşan, \& Clichici, 2013; Gutierrez, Prater, \& Holladay, 2014; Song, 2005).
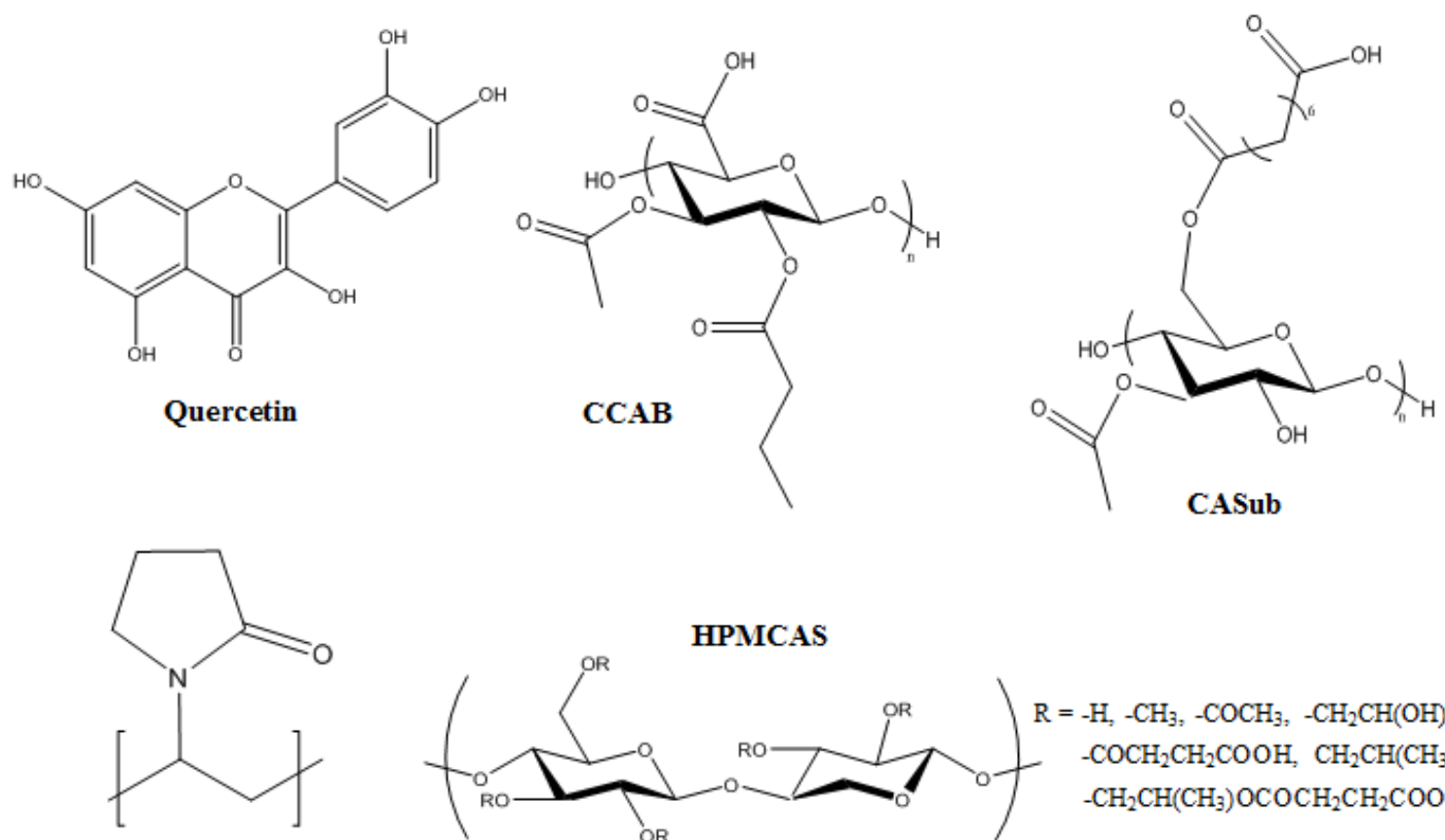

PVP

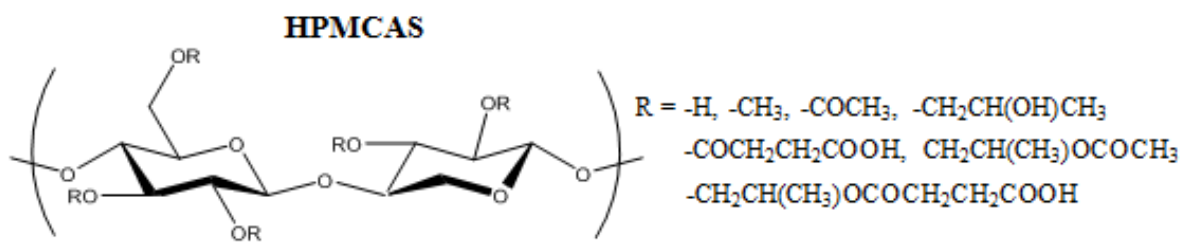

Figure 1. Chemical structures of $Q, C C A B, C A S u b, P V P$, and HPMCAS. The cellulosic structures are not meant to convey regioselective substitution; depictions of substituent location are merely for convenience and clarity of depiction.

Poor Q oral bioavailability severely limits its potential to benefit health. This low bioavailability is largely due to its crystallinity, and hence poor solubility (ranging from 2.15-7.7 $\mu \mathrm{g} / \mathrm{mL}$ at $25^{\circ} \mathrm{C}$ (Maria Rosaria Lauro et al., 2002; Srinivas, King, Howard, \& Monrad, 2010)) in the aqueous milieu of the gut lumen, as well as extensive metabolism and subsequent luminal efflux by gut epithelial cells (Phase-II and Phase-III xenobiotic metabolism, respectively). Improved Q solubility may increase bioavailability by increasing the amount available for absorption, and by saturating Phase-II and Phase-III metabolic enzymes; both effects are likely to result in increased net flux into circulation.

Many techniques have been employed to improve $\mathrm{Q}$ oral bioavailability, such as protein- or cellulose-based nanoparticles (Fang et al., 2011; Kakran, Sahoo, Li, \& Judeh, 2012; Sahu, Saraf, Kaur, \& Saraf, 2013), encapsulation (Dian et al., 2014), nanoemulsifying drug delivery systems (Tran, Guo, Song, \& Bruno), and ASD (Ilevbare, Liu, Edgar, \& Taylor, 2012; Li et al., 2013; Pereira et al., 2013). ASD preparation with polymer dispersants is an attractive way to stabilize the high energy, amorphous drug in a glassy polymeric matrix. Exposure of the ASD to the GI lumen not only provides supersaturated drug solutions, but also enhances permeation by 
increasing the drug concentration gradient across the enterocytes. Polymer selection is key for ASD performance because the dispersion must be miscible, with strong polymer-drug interactions (e.g. hydrogen bonding) for stability against crystallization (Pereira et al., 2013; Vasconcelos, Sarmento, \& Costa, 2007). Amphiphilic polymers possessing carboxylic acid functionality perform well in ASD due to strong polymer-drug interactions; their $\mathrm{pH}$ responsiveness is also valuable. At gastric $\mathrm{pH}$, the protonated form protects the drug and minimizes release, while deprotonation at near-neutral intestinal $\mathrm{pH}$ swells the polymer and triggers drug release (Li et al., 2013; Pereira et al., 2013). Cellulose derivatives are popular ASD polymers due to their generally benign nature and high $\mathrm{T}_{\mathrm{g}}$ values. CASub and cellulose acetate adipate propionate (CAAdP) were synthesized in the Edgar lab and show high promise for ASD (Li et al., 2013; Liu et al., 2014). Novel ASD polymers are needed to go beyond the performance of currently used polymers like PVP and HPMCAS that were not designed for ASD, and cellulosic polymers are ideal candidates due to their generally low toxicity and lack of oral bioavailability.

ASD has been only lightly explored for Q dissolution enhancement. Lauro et al. achieved slight dissolution enhancement using ASDs prepared with cross-linked sodium carboxymethylcellulose and sodium starch glycolate (Maria Rosaria Lauro et al., 2002). Similarly, Lauro et al. used spray dried dispersions with cellulose acetate trimellitate and cellulose acetate phthalate to improve Q release at pH 6.8 (M. R. Lauro, Maggi, Conte, De Simone, \& Aquino, 2005). Recently, several polymers were evaluated for their ability to improve $\mathrm{Q}$ dissolution in vitro (Li et al., 2013). HPMCAS afforded ASDs containing up to 50\% Q content; enhanced dissolution was obtained from 10\% Q ASDs, optimally in polymer blends containing $10 \%$ of the water-soluble (PVP) (Gupta, Kakumanu, \& Bansal, 2004; Konno, Handa, Alonzo, \& Taylor, 2008; Van den Mooter et al., 2001). Employing PVP in blends with other cellulosic polymers may generally enhance drug release, while retaining the excellent stabilization from the cellulosic polymer (Marks, Wegiel, Taylor, \& Edgar, 2014).

The objective of this study was to assess the performance of the novel polymer CASub for making Q ASDs and creating supersaturated Q solutions at physiological $\mathrm{pH}$, vs. crystalline Q as negative control and HPMCAS/Q ASD as positive control. We hypothesized that 1) CASub would provide enhanced solution concentration and preferable dissolution kinetics compared to HPMCAS, and 2) that blending CASub with PVP would further enhance Q dissolution.

\section{EXPERIMENTAL}

\subsection{Materials.}

Quercetin ( $\geq 95 \%$ by HPLC), epicatechin (EC) ( $\geq 90 \%$ by HPLC), and $\mathrm{KCl}$ (solid, anhydrous, $\geq$ 99\%) were purchased from Sigma-Aldrich. Cellulose acetate propionate (CAP-504-0.2; degree of substitution $(\mathrm{DS})$ (acetate) $=0.04, \mathrm{DS}$ (propionate) $=2.09 ; \mathrm{M}_{\mathrm{n}}=15,000$ ); CCAB; DS $($ butyrate $)=1.62, \mathrm{DS}($ acetate $)=0.06, \mathrm{DS}$ (carboxylic acid) $=0.28) ; \mathrm{Mw}=252,000)$ and cellulose acetate $(\mathrm{CA} 320 \mathrm{~S}, \mathrm{DS}$ (acetate) $\left.=1.82) \mathrm{M}_{\mathrm{n}}=50,000\right)$ were from Eastman Chemical Company. HPMCAS (wt \%: methoxyl 20-24\%, hydroxypropyl 5-9\%, acetyl 5-9\%, succinoyl 14-18\%; $\mathrm{Mw}=18,000)$ was from Shin-Etsu Chemical Co., Ltd. Chemical structures of ASD polymers used (HPMCAS, PVP, CCAB, and CASub) are provided in Fig 1. Acetonitrile (ACN, HPLC-grade), methylene chloride (HPLC-grade), tetrahydrofuran (THF), reagent ethanol, sodium phosphate monobasic, and sodium hydroxide $(\mathrm{NaOH})$ were purchased from Fisher Scientific and used as received. $\mathrm{HCl}(12.1 \mathrm{M})$ was obtained from Macron Chemicals. Suberic 
acid, adipic acid, methyl ethyl ketone (MEK), p-toluenesulfonic acid (PTSA), triethylamine $\left(\mathrm{Et}_{3} \mathrm{~N}\right)$, and oxalyl chloride were purchased from ACROS Organics. 1,3-Dimethyl-2imidazolidinone (DMI) was purchased from ACROS Organics and dried over $4 \AA$ molecular sieves. Water was purified by reverse osmosis and ion exchange using a Barnstead RO pure ST (Barnstead/Thermolyne) purification system. LCMS grade ACN, water and formic acid were obtained from VWR.

\subsection{Synthesis of CASub}

CASub was synthesized as previously reported (Liu et al., 2014). See Supplemental Information for full details.

\subsection{Preparation of ASDs via spray drying.}

Supplemental Information contains a full description of preparation of ASDs containing Q. Our convention for naming treatments is to list the \% polymer(s), with the remainder being Q. For example, $10 \% \mathrm{Q} / 90 \% \mathrm{CCAB}$ is referred to as $90 \mathrm{CCAB}$ in the text, figures and tables. ASDs prepared were: $90 \mathrm{CCAB}, 75 \mathrm{CCAB}, 50 \mathrm{CCAB}, 10 \mathrm{PVP}: 80 \mathrm{CCAB}, 20 \mathrm{PVP}: 70 \mathrm{CCAB}, 90$ HPMCAS, 90 CASub, 10 PVP:80 CASub, 20 PVP:70 CASub. ASD Q content was confirmed by UPLC to be within $4 \%$ of targeted value in every case (Supplementary Table 1).

\subsection{ASD Characterization.}

2.4.1. XRD, DSC and FT-IR.

X-ray powder diffraction, DSC and FTIR were performed as described in in Supplementary Information.

\subsubsection{Determination of crystalline and amorphous $Q$ solubility.}

Crystalline solubility: An excess of crystalline $\mathrm{Q}$ was added to $15 \mathrm{~mL} \mathrm{pH} 6.8$ buffer solution, or $\mathrm{pH} 2.5$ solution. Solutions were equilibrated at $37{ }^{\circ} \mathrm{C} / 48 \mathrm{~h}$ with constant agitation, protecting them from light.

Amorphous solubility: Supersaturated Q solutions were prepared by adding a specific amount of Q stock solution $(4 \mathrm{mg} / \mathrm{mL}$ in $\mathrm{MeOH})$ to $15 \mathrm{~mL}$ buffer at $37{ }^{\circ} \mathrm{C}$. Potassium phosphate buffer (100 mM, pH 6.8) with $100 \mu \mathrm{g} / \mathrm{mL}$ PVP (Kollidon ${ }^{\circledR} 12$ PF); or acidified distilled water (pH 2.5) with $100 \mu \mathrm{g} / \mathrm{mL}$ PVP was used. The polymer was added to inhibit drug crystallization during the experiment and accurately determine the "amorphous solubility" of Q. Total Q concentration of was $80 \mu \mathrm{g} / \mathrm{ml}$.

Crystalline and supersaturated solutions were centrifuged at 35,000 rpm $(274,356 x \mathrm{~g})$ for 30 minutes to separate the precipitated drug phase using an Optima L-100 XP ultracentrifuge equipped with Swinging-Bucket Rotor SW 41 Ti (Beckman Coulter, Inc., Brea, CA). Following centrifugation, the supernatant was collected, diluted (1:1) with methanol, and the final concentrations were measured by HPLC using an Agilent HPLC 1260 Infinity system (Agilent Technologies, CA, USA) with an Agilent Eclipse plus C18, 4.6 × $250 \mathrm{~mm}, 5 \mu \mathrm{m}$ analytical column (Agilent technologies, CA, USA). Full HPLC methodological details are presented in Supplementary Information.

\subsection{In vitro dissolution.}


166 In vitro dissolution was performed under non-sink conditions to evaluate Q dissolution 167 concentrations and kinetics achieved via ASD delivery, compared to crystalline Q alone, under 168 conditions similar to normal human gastrointestinal conditions. Full details in Supplemental 169 Information.

\subsection{UPLC-MS/MS.}

Internal standard solution $(50 \mu \mathrm{L}, 0.8 \mathrm{mg} / \mathrm{mL} \mathrm{EC}$ in ethanol), $50 \mu \mathrm{L}$ diluted dissolution supernatant, and $900 \mu \mathrm{L}$ of $0.1 \%$ formic acid in $80 \%$ water/20\% 80:20 ACN/THF were added to Waters UPLC vials (Milford, MA) and mixed. Analysis of Q content within ASDs and subsequent in vitro dissolution solution concentrations were performed by UPLC-MS/MS (method details in Supplemental Information).

\subsection{Data Analysis and Statistics.}

Dissolution results are reported as soluble Q vs. time. Dissolution kinetic parameters were determined from these data. Pseudo-pharmacokinetic parameters (area under the concentrationtime curve: AUC; maximal observed solution concentration: $\mathrm{C}_{\mathrm{MAX}}$; time at which maximal solution concentration was observed: $\mathrm{T}_{\mathrm{MAX}}$ ) were computed from $\mathrm{Q}$ concentration/time data using standard plugins for Microsoft Excel (Redmond, WA). Prism v. 6.0d (GraphPad, la Jolla, CA) was used to perform statistical comparisons. Dixon's Q-test $(\alpha=0.05)$ was utilized to identify and exclude any outliers as necessary. Significant differences in dissolution parameters between treatments were determined using one-way ANOVA with Tukey's HSD post hoc test performed on treatment means. Significance was defined a priori as $\mathrm{P}<0.05$.

\section{RESULTS AND DISCUSSION}

Three promising carboxylated cellulose derivatives were selected for ASD preparation in order to increase Q apparent solubility (Fig. 1). CCAB is a new commercial carboxylated cellulose ester (Buchanan et al., 2014), and HPMCAS is a cellulose ether-ester that is in commercial use as an efficient ASD polymer (Li et al., 2013). CASub was recently designed by the Edgar and Taylor groups as a promising ASD polymer and crystal growth inhibitor (Liu et al., 2014). The above-mentioned polymers may not only stabilize amorphous $\mathrm{Q}$ in the solid state and prevent recrystallization after release, but also provide targeted $\mathrm{pH}$-controlled release to the small intestine where Q absorption occurs. We also investigated blends of CASub and CCAB with PVP to promote enhanced Q dissolution, since Q and thus its ASDs with polymers like CASub and $\mathrm{CCAB}(\delta=22.66$ and 24.44 , respectively) are rather hydrophobic. ASD drug concentration influences both the practicality of the method and ASD performance, so to explore this influence, $\mathrm{Q}$ concentration in the ASD was varied $(10 \%, 25 \%$ and $50 \% \mathrm{Q}(\mathrm{w} / \mathrm{w}))$, using readily available commercial $\mathrm{CCAB}(90 \mathrm{CCAB}, 75 \mathrm{CCAB}, 50 \mathrm{CCAB})$ as the test system. To compare effectiveness of novel polymers and polymer blends, dissolution profiles were compared against commercial polymers HPMCAS and CCAB.

\subsection{Solid State Characterization of $Q$ loaded ASDs.}




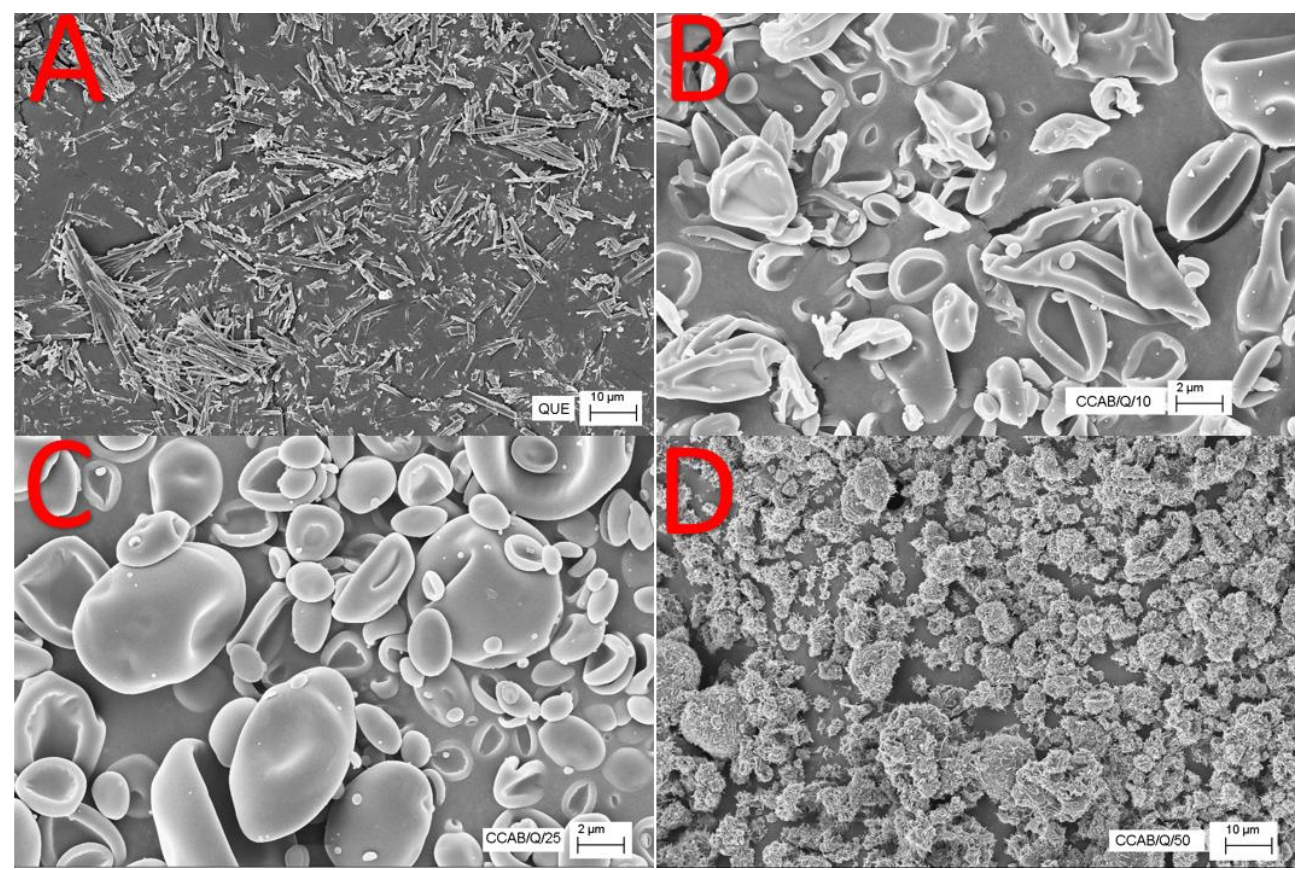

Figure 2. SEM images (mag. 10X) for crystalline $Q(A), 90 C C A B(B), 75$ $C C A B(C)$, and $50 C C A B(D)$ are shown to illustrate particle size range (1-3 $\mu \mathrm{m})$ and morphology.

Morphology was examined by SEM (Fig. 2; other SEM images see Supplemental Fig. S1-4). All ASD particles exhibited a smooth surface, indicating absence of evident crystals, except for 50\% $\mathrm{Q}$ loaded CCAB that appeared to contain Q crystals. Particles have corrugated morphology, crushed, indented and collapsed, typical of polysaccharide-based spray-dried ASDs.

XRD was used to determine whether dispersions were amorphous. All XRD spectra showed only amorphous haloes (Fig. 3), except those of crystalline Q and 50\% Q in CCAB (Fig. 3A). XRD data strongly support the amorphous nature of these dispersions except for $50 \mathrm{CCAB}$, which was therefore excluded from further testing. XRD spectra for 90 HPMCAS and 90 CASub are presented in Supplemental Fig. 5.

DSC was used to further examine dispersion morphology of $\mathrm{Q}$ and polymers; data from

221 CASub and CCAB are presented in Fig. 4. Although crystalline Q melts at $326{ }^{\circ} \mathrm{C}$, DSC scans were kept $\leq 185^{\circ} \mathrm{C}$, due to concerns about potential crosslinking of these polymers (containing both $\mathrm{OH}$ and $\mathrm{CO}_{2} \mathrm{H}$ groups) above that temperature (Posey-Dowty et al., 2007; Shelton et al., 2009). For ASDs a glass transition $\left(\mathrm{T}_{\mathrm{g}}\right)$ temperature lower than that of the pure polymer is expected. Polymer $\mathrm{T}_{\mathrm{g}}$ values are $175^{\circ} \mathrm{C}(\mathrm{PVP}), 144^{\circ} \mathrm{C}(\mathrm{CASub})$ and $134^{\circ} \mathrm{C}(\mathrm{CCAB})$. ASDs $(10 \%$ $\mathrm{Q})$ all had lower $\mathrm{T}_{\mathrm{g}}$ values than the corresponding pure polymer, indicating that $\mathrm{Q}$ acted as a plasticizer. Since Q melts higher than the decomposition temperatures of several of our polymers, absence of $\mathrm{Q} \mathrm{T}_{\mathrm{m}}$ and $\mathrm{T}_{\mathrm{c}}$ in the ASDs could not be confirmed. DSC $\mathrm{T}_{\mathrm{g}}$ values along with the XRD data were sufficient to confirm the amorphous character of $\mathrm{Q}$ in these dispersions. DSC heating curves for all other dispersions are presented in Supplemental Fig. 6.

FT-IR spectra are presented and discussed in the Supplemental Information. 

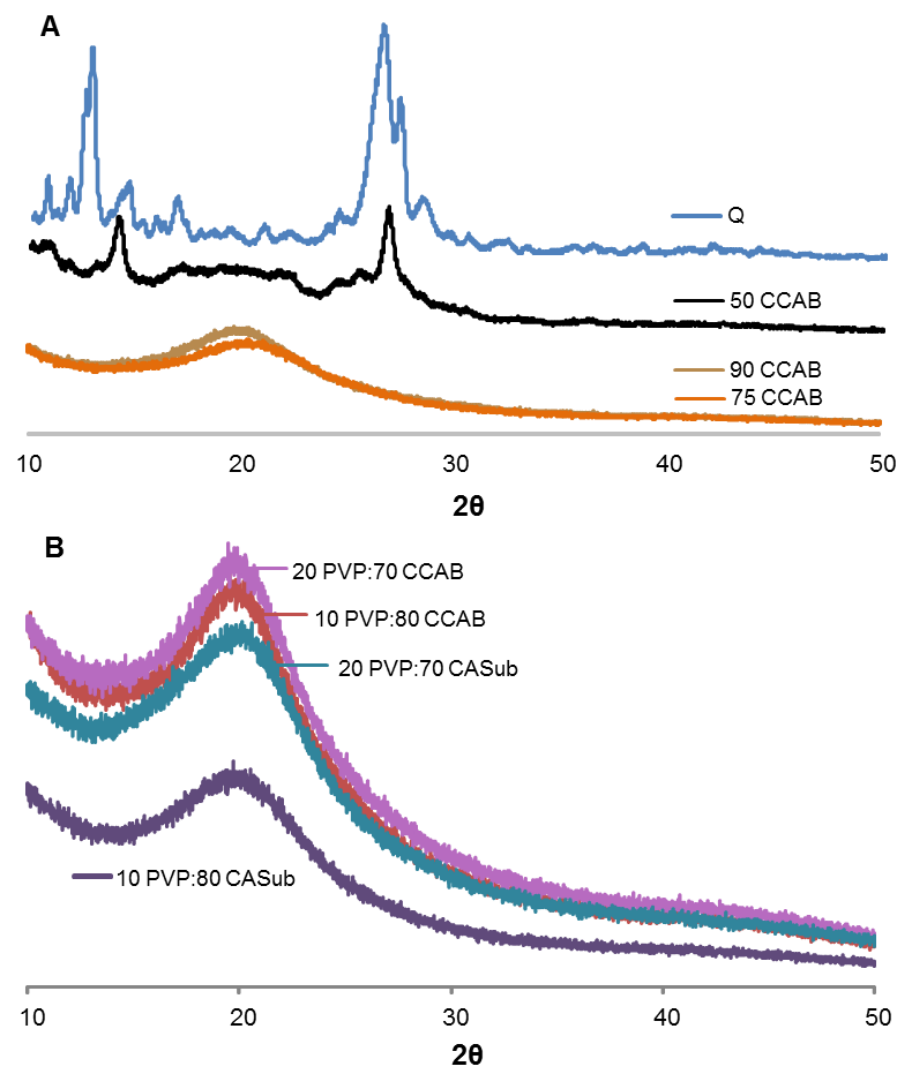

Figure 3. XRD spectra of $Q, 50 C C A B, 75 C C A B$, and $90 C C A B$ (A) as well as the PVP blends with both CCAB and CASub (B).

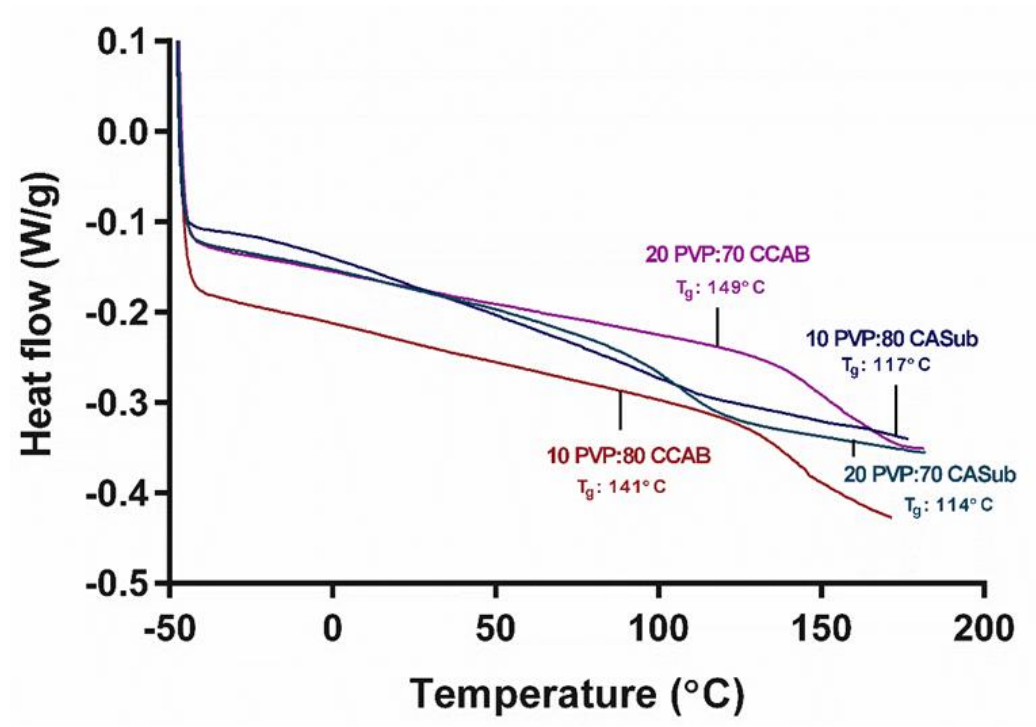

Figure 4. DSC second heating curves of Q-loaded ASDs of CASub, $C C A B$, and their respective blends containing $20 \%$ PVP.

239 We attempted to confirm the crystalline solubility and measure Q amorphous solubility (Table 240 1). Amorphous forms of compounds have a maximum apparent solubility, which represents the 
maximum amount of free drug achievable in solution, termed amorphous solubility (Ilevbare \& Taylor, 2013). The experimental amorphous solubility can be measured by creating supersaturated solutions by a solvent-shift method, and then measuring the concentration of drug in the supernatant. However, for compounds that are fast crystallizers (like Q), it is necessary to add a small amount of polymer (in this case PVP) to stabilize the supersaturated solution, inhibiting crystallization, and permitting accurate measurement of amorphous solubility. It was impossible to measure Q amorphous solubility in the absence of polymer, because the drug crystallized upon contact with the aqueous solution. We measured Q crystalline solubility at low (2.5) $\mathrm{pH}$, where $\mathrm{Q}$ is un-ionized, and at $\mathrm{pH} 6.8$ where it is partially ionized. Q amorphous solubility was also measured at both $\mathrm{pH}$ values. Crystalline solubility values were determined in the absence and presence of PVP, while amorphous solubility was determined only in the presence of PVP. Amorphous solubility was significantly higher than its crystalline counterpart at both $\mathrm{pH}$ values. Q crystalline solubility was similar to literature reports, and amorphous solubility appears to be at least $31 \mu \mathrm{g} / \mathrm{mL}$ at small intestine $\mathrm{pH}$.

Table 1. Crystalline and amorphous solubility of Q in varying dissolution medium (values are mean \pm SEM).

\begin{tabular}{ccc} 
Medium & $\begin{array}{c}\text { Crystalline solubility } \\
(\boldsymbol{\mu} \mathbf{g} / \mathbf{m l})\end{array}$ & $\begin{array}{c}\text { Amorphous solubility } \\
(\boldsymbol{\mu g} / \mathbf{m l})\end{array}$ \\
\hline Acidified water $(\mathrm{pH} 2.5)^{\mathrm{a}}$ & $0.64 \pm 0.11$ & $\mathrm{~N}^{\mathrm{b}}$ \\
Acidified water $(\mathrm{pH} 2.5)^{\mathrm{a}}+100 \mu \mathrm{g} / \mathrm{ml} \mathrm{PVP}$ & $1.03 \pm 0.20$ & $23.48 \pm 0.06$ \\
$100 \mathrm{mM}$ potassium phosphate buffer $(\mathrm{pH} 6.8)$ & $1.03 \pm 0.08$ & $\mathrm{~N}^{\mathrm{b}} \mathrm{A}^{\mathrm{b}}$ \\
$100 \mathrm{mM}$ potassium phosphate buffer $(\mathrm{pH} 6.8)+100$ & N/A $^{\mathrm{c}}$ & $31.29 \pm 1.80$ \\
\hline
\end{tabular}

\footnotetext{
${ }^{a}$ Acidified with phosphoric acid

${ }^{\mathrm{b}}$ Measurement not performed in absence of polymer due to fast $\mathrm{Q}$ crystallization upon contact with the solution

${ }^{\mathrm{c}}$ Measurement not performed
} in Fig. 5A, both 10\% and 25\% Q loaded CCAB ASDs (90 CCAB, 25 CCAB) effectively protected $\mathrm{Q}$ from release at acidic $\mathrm{pH}$; indeed, solution concentrations were significantly lower than that from crystalline $\mathrm{Q}$ alone. At the neutral $\mathrm{pH}$ that mimics the small intestine, release from the 10\% Q dispersion (90 CCAB, Fig. 5B) reached much higher solution concentrations than from the $25 \%$ Q ASD (75 CCAB) or from crystalline Q alone. This is predictable given the quite hydrophobic nature of Q (more Q in the ASD makes it more hydrophobic, slowing drug release), and is consistent with our results with CAAdP and other polymers ( $\mathrm{Li}$ et al., 2013). As a result we chose to compare subsequent ASD formulations with different polymers using $10 \%$ Q in each 

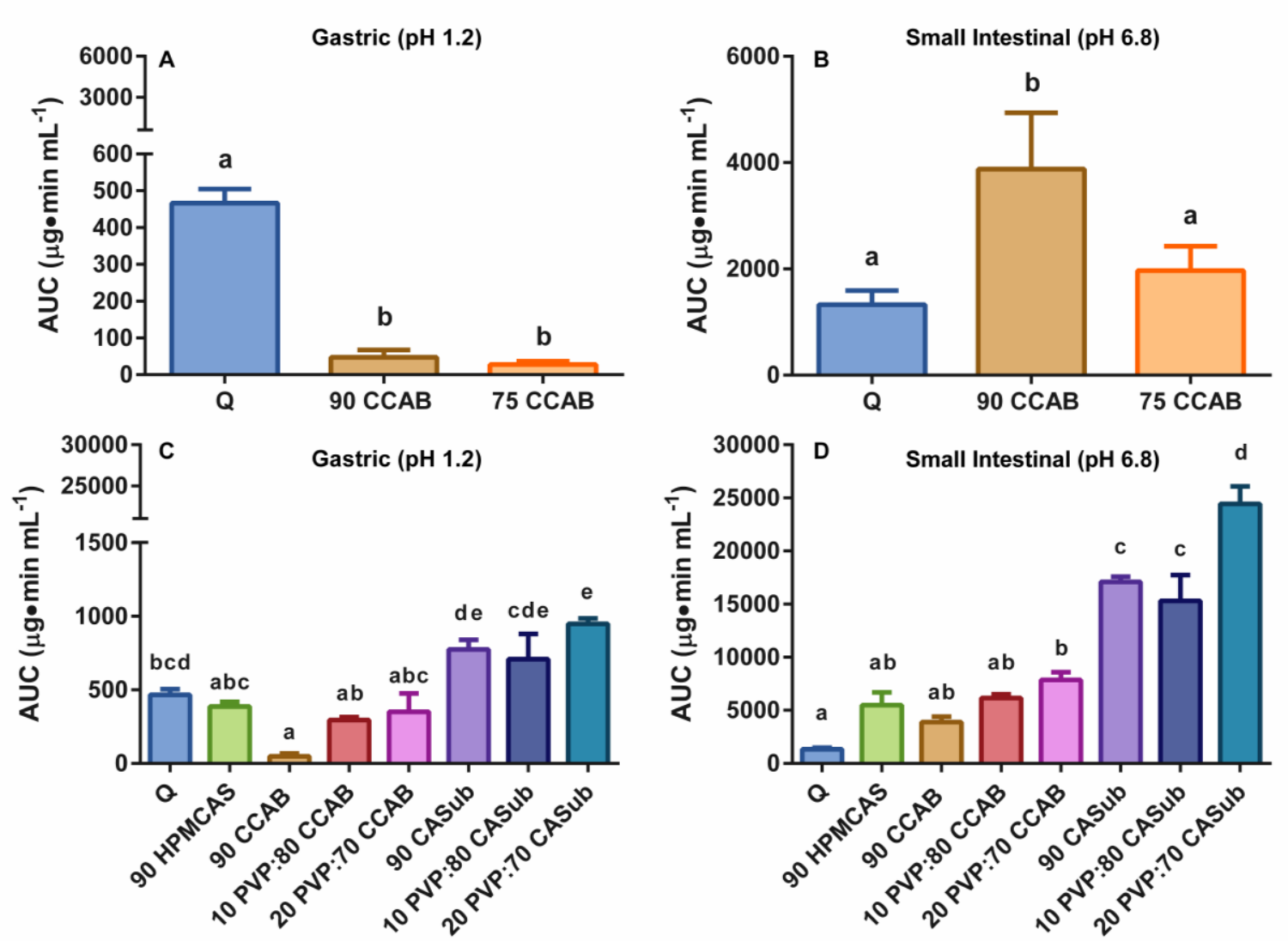

Figure 5. Dissolution area under the curve $(A U C)$ values for all treatments. $Q$ compared to $90 C C A B$ and 75:25 CCAB is given for both gastric $\mathrm{pH}(A)$ and intestinal $\mathrm{pH}(B) . Q$ compared to all treatments that employed $10 \% Q$ loads are shown for both gastric $\mathrm{pH}(\mathrm{C})$ and intestinal $\mathrm{pH}(D)$. Values are mean \pm SEM. Different letters above each bar represent statistically significant differences in AUC between treatments $(p<0.05)$ by 1 -way ANOVA with Tukey's post hoc test.

\subsubsection{Simulated gastric conditions $(p H=1.2)$}

Crystalline Q and all Q ASDs had extremely low dissolution in the gastric environment (Fig. 5C, Supplemental Table 2). This was expected due to the inherent properties of crystalline $\mathrm{Q}$, and of carboxylated cellulose derivatives (protonated, thus neutral/poorly soluble at acidic $\mathrm{pH}$ ). Maximum low pH Q release was from 10\% Q dispersed in 20\% PVP:70\% CASub, reaching only a mean $\mathrm{C}_{\mathrm{MAX}}$ of $13.4 \mu \mathrm{g} / \mathrm{mL}$ and AUC of $972 \mu \mathrm{g} \cdot \mathrm{min} / \mathrm{mL}$. All other polymers were relatively successful in preventing Q release at gastric $\mathrm{pH}$. Even the AUC from 20\%PVP:70\% CASub at $\mathrm{pH} 1.2$ was quite low compared to observed AUC values for any treatment at $\mathrm{pH} 6.8$.

\subsubsection{Simulated small intestinal conditions $(p H=6.8)$}

Dissolution experiments were performed at $\mathrm{pH} 6.8$ to mimic the small intestine, and under nonsink conditions where $\geq 31$-fold supersaturation would be achieved if all drug dissolved, in order to permit observation and quantification of the supersaturation expected from ASDs. AUC measurements for all formulations are presented in Fig 5D; AUC, $\mathrm{C}_{\mathrm{MAX}}$, and $\mathrm{T}_{\mathrm{MAX}}$ values are summarized in Table 2. All ASDs examined provided some degree of supersaturation vs. Q alone. ASDs prepared with only HPMCAS or CCAB provided only modest (non-significant) 
improvements in Q solution concentrations; this is particularly interesting for HPMCAS, which has some solubility in water (ca. $23 \mathrm{mg} / \mathrm{mL}$ ) (Li et al., 2013). In contrast, CCAB blended with PVP, and CASub either by itself or blended with PVP all gave very significantly supersaturated Q solutions. Optimum Q dissolution was observed with CASub and PVP:CASub blends. Overall, 10\% Q dispersed in 20 PVP:70 CASub provided the most significant enhancement ( $\mathrm{p}<$ 0.05 ) in $\mathrm{Q}$ apparent solution concentration, with an 18-fold increase in AUC compared to crystalline $\mathrm{Q}(\sim 24,400 \mathrm{vs.} 1,330 \mu \mathrm{g} \cdot \mathrm{min} / \mathrm{mL}$, respectively). This ASD was also able to produce the highest maximum solution concentration $\left(\mathrm{C}_{\mathrm{MAX}}=78.3 \mu \mathrm{g} / \mathrm{mL}\right)$ over the course of $8 \mathrm{~h}$ at $\mathrm{pH}$ 6.8. This average $C_{\operatorname{MAX}}$ value is 12.7 -fold higher than the average $\mathrm{C}_{\mathrm{MAX}}$ value attained by crystalline Q $(6.16 \mu \mathrm{g} / \mathrm{mL})$. The degrees of enhancement of Q solution concentrations achieved are comparable to those achieved from different amorphous matrices by others (Fujimori et al., 2015) and in our previous work (Li et al., 2013).

Table 2. Pseudo-pharmacokinetic parameters of Q at intestinal $\mathrm{pH}$ (6.8).

\begin{tabular}{|c|c|c|c|}
\hline ASD Formulation & $\begin{array}{c}\mathrm{AUC}^{\mathrm{a}} \\
(\mu \mathrm{g} \mathrm{min} / \mathrm{mL})\end{array}$ & $\begin{array}{c}C_{\text {MAX }}{ }^{a} \\
(\mu \mathrm{g} / \mathrm{mL})\end{array}$ & $\begin{array}{l}\mathbf{T}_{\text {MAx }}{ }^{2} \\
\text { (min) }\end{array}$ \\
\hline Q & $1330 \pm 133$ & $6.16 \pm 0.141$ & 120 \\
\hline $90 \mathrm{CCAB}$ & $3880 \pm 529$ & $18.4 \pm 2.15$ & 60 \\
\hline $75 \mathrm{CCAB}$ & $1940 \pm 254$ & $7.16 \pm 1.49$ & 60 \\
\hline $50 \mathrm{CCAB}$ & $2550 \pm 137$ & $8.21 \pm 0.760$ & $\mathrm{~N} / \mathrm{A}^{\mathrm{b}}$ \\
\hline 10 PVP:80 CCAB & $6210 \pm 382$ & $20.0 \pm 1.33$ & 480 \\
\hline 20 PVP:70 CCAB & $7850 \pm 733$ & $27.4 \pm 3.03$ & 420 \\
\hline $90 \mathrm{HPMCAS}^{\mathrm{c}}$ & $5480 \pm 1210$ & $64.1 \pm 19.7$ & 30 \\
\hline $90 \mathrm{CASub}^{\mathrm{c}}$ & $17100 \pm 485$ & $48.7 \pm 1.74$ & $\mathrm{~N} / \mathrm{A}^{\mathrm{b}}$ \\
\hline 10 PVP:80 CASub ${ }^{c}$ & $15300 \pm 2434$ & $50.4 \pm 4.58$ & 90 \\
\hline 20 PVP:70 CASub ${ }^{c}$ & $24400 \pm 1640$ & $78.3 \pm 7.12$ & $\mathrm{~N} / \mathrm{A}^{\mathrm{b}}$ \\
\hline
\end{tabular}

Blending with the miscible and hydrophilic PVP enhanced Q release from both CCAB and CASub as anticipated. The concern was whether the lower concentration of the effective crystallization inhibitors CCAB (Marks, J.A., Nichols, B.L.B., Edgar, K.J. manuscript in preparation) or CASub (Ilevbare, Liu, Edgar, \& Taylor, 2013)) would lead to loss of Q solution concentration due to crystallization. This does not appear to have been the case, and the blending approach was effective at synergistically combining PVP release properties with CASub/CCAB crystallization inhibition properties. Our data suggest that combination of hydrophobic (CCAB and CASub) and hydrophilic (PVP) polymers appears to provide relative protection from gastric conditions, enhanced release profile, and prevent $\mathrm{Q}$ recrystallization once in solution.

Polymer solubility parameters, presented in Table 3, are important, albeit imperfect, 311 predictors of whether the polymer has the right hydrophobic/hydrophilic balance (they do not have the ability to discriminate between ionized and un-ionized carboxyl, which for this purpose is an important flaw). Solubility parameter calculations were performed as previously reported (Fedors, 1974; Liu et al., 2014) and can be found in Supplemental Information. Dissolution curves obtained with different ASDs are shown in Fig. 6 (pH 6.8) and Supplemental Fig. 8 (pH 1.2). Higher solubility parameters indicate greater polymer hydrophilicity, therefore the polymers arranged by decreasing hydrophobicity are PVP $<$ CCAB $<$ CASub $<$ HPMCAS. The correlation between $\mathrm{Q}$ release and polymer solubility parameter is rather weak; this has been 
observed also in other polymer-drug systems (Mosquera-Giraldo, L.I., Meng, X., Edgar, K.J., Slipchenko, L.V., Taylor, L.S., manuscript under review). Within more confined data sets, solubility parameters can have predictive value; thus, addition of the quite hydrophilic PVP enhances release from the more hydrophobic CCAB and CASub matrices in predictable fashion. Based on solubility parameters alone, for example, the low Q solution concentration obtained from the 10\% Q ASD in CCAB ASD was unexpected; a maximum Q solution concentration of only $18.4 \mu \mathrm{g} / \mathrm{mL}$ (Fig. 6B) was attained. Lower concentration with CCAB than with CASub was surprising since $\mathrm{CCAB}$ has higher calculated solubility parameter than CASub. CASub was especially effective at enhancing Q solution concentration, reaching a maximum of $48.7 \mu \mathrm{g} / \mathrm{mL}$ within the first hour of dissolution (Fig. 6B). This is fully consistent with the known excellence of CASub as a crystallization inhibitor (Ilevbare et al., 2013). When blended with 10\% PVP, release and thus solution concentration did not improve noticeably $(50.4 \mu \mathrm{g} / \mathrm{mL})$, but with $20 \%$ PVP in the ASD (20 PVP:70 CASub), solution concentration improved markedly to $78.3 \mu \mathrm{g} / \mathrm{mL}$ (Fig. 6D). HPMCAS, the most hydrophobic polymer in the set of polymers investigated, provided interesting Q ASD behavior. Release from the HPMCAS ASD was very rapid, reaching an average solution concentration of $64.1 \mu \mathrm{g} / \mathrm{mL}$ (Fig. 6B) within $30 \mathrm{~min}$. This solution was clear, but quickly became cloudy, and measured solution concentration quickly dropped off, indicating that HPMCAS was ineffective at preventing Q crystallization from supersaturated solution.

Table 3. Hildebrand solubility parameters of polymers used to prepare Q ASDs.

\begin{tabular}{cc}
\hline Polymer & $\begin{array}{c}\text { Solubility parameter, } \boldsymbol{\delta} \\
\left(\mathbf{M P a}^{\mathbf{1 / 2}}\right)\end{array}$ \\
\hline PVP & 28.39 \\
CCAB & 24.44 \\
CASub (DS 0.9) & 22.66 \\
HPMCAS & 22.42 \\
\hline
\end{tabular}



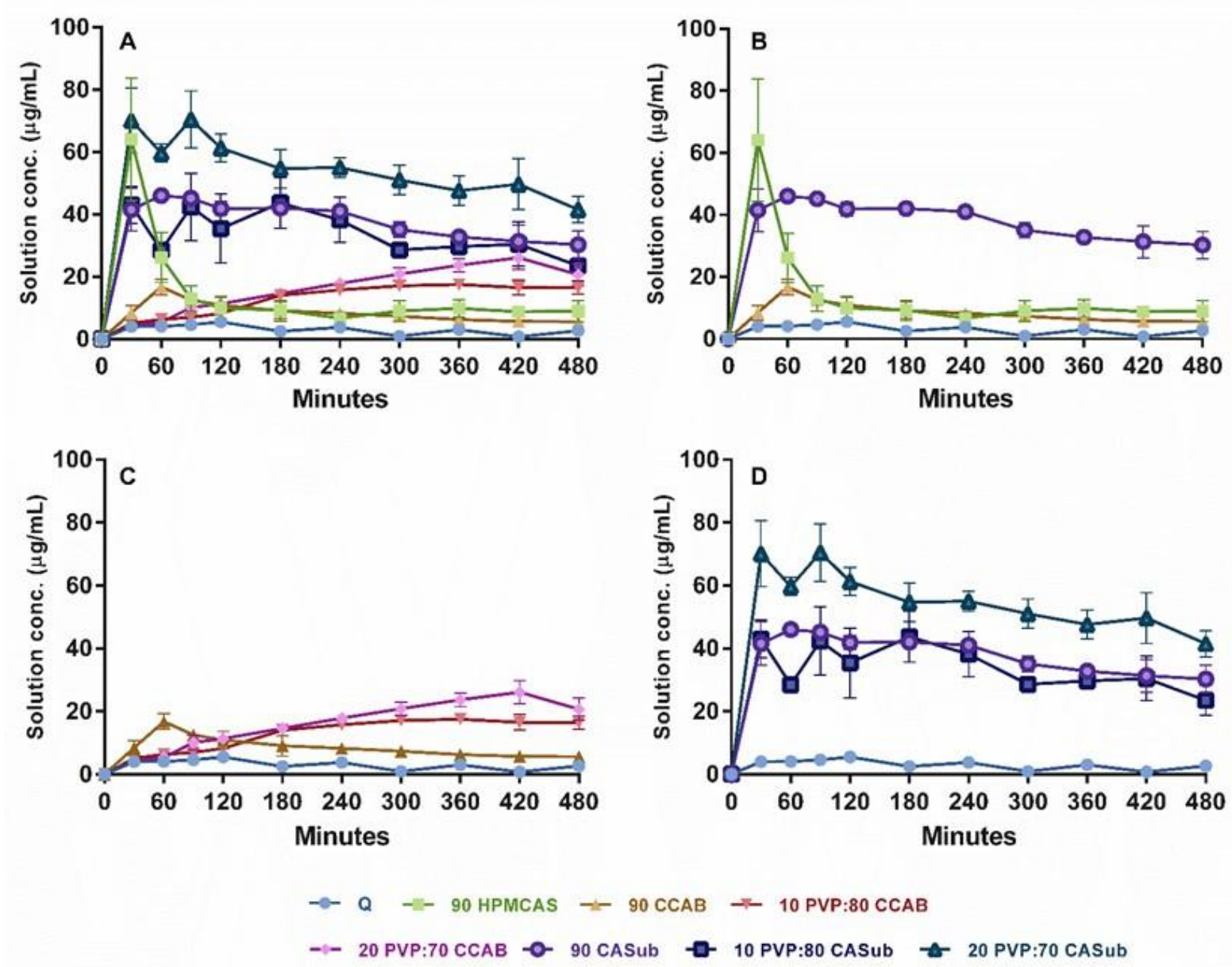

Figure 6. Average solution concentrations of $Q$ (mean $\pm S E M, n=3-4)$ plotted over time at $p H 6.8$ for all treatments $(A), 10 \% Q$ loaded ASDs only shown for comparison of dissolution properties for each polymer utilized $(B)$, the impact of PVP blending with CCAB $(C)$ and CASub $(D)$. All graphs contain crystalline $Q$ as a control for comparison. Values are mean \pm SEM.

341 3.4. Summary of results

342 Amorphous solid dispersions at $10 \mathrm{wt} \%$ Q were prepared using the cellulose esters CCAB, 343 HPMCAS, and CASub. Q was also amorphous at $25 \%$ in CCAB, but was not fully amorphous at $34450 \%$ Q in CCAB. All ASDs of Q in these carboxyl-containing cellulose esters protect effectively 345 against $\mathrm{Q}$ release at fasting gastric $\mathrm{pH}$, giving substantially lower Q concentrations than from $\mathrm{Q}$ 346 without polymer. In contrast, all three cellulose esters provide supersaturated solutions of Q at small intestinal $\mathrm{pH}$, at which $\mathrm{pH}$ most of the carboxylic acids are ionized. The degree of supersaturation from CCAB-only dispersions was slight, due in part to ineffective drug release, while release from HPMCAS ASD was rapid, achieving substantial supersaturation. However HPMCAS proved to be a poor inhibitor of Q recrystallization from supersaturated solutions, resulting in rapid de-supersaturation. CASub on the other hand provided both substantial Q release at $\mathrm{pH}$ 6.8, and stable, high supersaturation. Furthermore, incorporation of the more hydrophilic and water-soluble PVP into ASD blends was effective, enhancing release from $\mathrm{CCAB}$ at $10 \% \mathrm{PVP}$, and significantly enhancing release and supersaturation from CASub ASDs at 20\% PVP. Thus CASub and its blends with PVP are highly effective polymers for enhancing 
Q solution concentration in vitro, and provide a promising opportunity for increasing Q bioaccessibility and bioavailability in vivo.

\section{CONCLUSIONS}

Overall these results confirm our hypotheses, and significantly illuminate structureproperty relationships of ASD polymers. We can term polymer properties like sufficiently high $\mathrm{T}_{\mathrm{g}}\left(50^{\circ} \mathrm{C}\right.$ or more above the highest likely ambient temperature) and solubility parameters within an effective range (hydrophobic enough to interact with hydrophobic drugs, hydrophilic enough to release them) as necessary but clearly not sufficient polymer properties for effectiveness in ASD. This work further confirms the value of the $\mathrm{pH}$-responsive carboxylic acid functional group in providing neutral $\mathrm{pH}$ release as well as desirable specific polymer-drug interactions, but this parameter alone is also insufficient to fully predict success or failure. This work also provides a further example of the value of polymer blends for achieving performance levels (in this case both release and crystallization inhibition) that would be difficult to achieve by ASD of drug, in this case Q, with a single polymer. More detailed study of expanded sets of polymers is necessary to further sort out the structural features required for effective ASD.

The results of this study, particularly with CASub, warrant in vivo investigation of Q ASDs as method for increasing Q bioavailability upon oral administration. They predict that significant supersaturation should be achievable in vivo; it will be of great interest to see whether this results in higher permeation in vivo, and in saturation of metabolic enzymes, thereby providing enhanced bioavailability, particularly of the unmetabolized native Q. If successful, such an ASD approach should enable animal and human in vivo bioavailability enhancement studies, and provide predictable absorbed doses that will enable informative dose-response studies, thus leading to exploration of whether the potential health benefits of $\mathrm{Q}$ can be realized in humans.

\section{ACKNOWLEDGEMENTS}

\subsection{Acknowledgements}

Funding for this research was provided by the Institute for Critical Technologies and Applied Sciences (ICTAS) at Virginia Tech (VT), via a Junior Faculty Collaborative grant to Drs. Neilson and Edgar. We thank Eastman Chemical Company for their kind donation of CA, CAP, and CCAB. We thank Shin-Etsu Chemical Co., Ltd. for their kind donation of HPMCAS. We are grateful to the VT Macromolecules Innovation Institute for their support, and to VT ICTAS for their material and facilities support. BLBN thanks the National Science Foundation for financial support through grant number DMR-130827. We also wish to thank Steve McCartney (Nanoscale Characterization and Fabrication Laboratory, Virginia Tech) for his help in taking SEM images, and Ann Norris (Department of Sustainable Biomaterials, Virginia Tech) for XRD analyses.

\section{REFERENCES}

Buchanan, C. M., Buchanan, N. L., Carty, S. N., Kuo, C. M., Lambert, J. L., Malcolm, M.O., Posey-Dowty, J. D., Watterson, T.L. Wood, M.D., Lindblad, M. S. US Patent 9,040,683 (2014). Cellulose interpolymers and methods of oxidation. 
Caridi, D., Trenerry, V. C., Rochfort, S., Duong, S., Laugher, D., \& Jones, R. (2007). Profiling and quantifying quercetin glucosides in onion (Allium cepa L.) varieties using capillary zone electrophoresis and high performance liquid chromatography. Food Chemistry, 105(2), 691-699.

Chiş, I. C., Baltaru, D., Maier, M., Mureşan, A., \& Clichici, S. (2013). Effects of quercetin and chronic (training) exercise on oxidative stress status in animals with streptozotocininduced diabetes. Bulletin of University of Agricultural Sciences and Veterinary Medicine Cluj-Napoca. Veterinary Medicine, 70(1), 31-39.

Dian, L., Yu, E., Chen, X., Wen, X., Zhang, Z., Qin, L., Wang, Q., Li, G., Wu, C. (2014). Enhancing oral bioavailability of quercetin using novel soluplus polymeric micelles. Nanoscale Research Letters, 9(1), 1-11.

Fang, R., Jing, H., Chai, Z., Zhao, G., Stoll, S., Ren, F., Liu, F., Leng, X. (2011). Design and characterization of protein-quercetin bioactive nanoparticles. Journal of Nanobiotechnology, 9(1), 19.

Fedors, R. F. (1974). A method for estimating both the solubility parameters and molar volumes of liquids. Polymer Engineering \& Science, 14(2), 147-154.

Firdous, A. B., Sharmila, G., Balakrishnan, S., RajaSingh, P., Suganya, S., Srinivasan, N., \& Arunakaran, J. (2014). Quercetin, a natural dietary flavonoid, acts as a chemopreventive agent against prostate cancer in an in vivo model by inhibiting the EGFR signaling pathway. Food \& Function, 5(10), 2632-2645.

Fujimori, M., Kadota, K., Shimono, K., Shirakawa, Y., Sato, H., \& Tozuka, Y. (2015). Enhanced solubility of quercetin by forming composite particles with transglycosylated materials. Journal of Food Engineering, 149, 248-254.

Gupta, P., Kakumanu, V. K., \& Bansal, A. K. (2004). Stability and Solubility of Celecoxib-PVP Amorphous Dispersions: A Molecular Perspective. Pharmaceutical Research, 21(10), 1762-1769.

Gutierrez, J. C., Prater, M. R., \& Holladay, S. D. (2014). Quercetin supplementation reduces maternal hyperglycemia in a type 2 diabetes mellitus mouse model. Annual Research \& Review in Biology, 4(1), 306-311.

Ilevbare, G. A., Liu, H., Edgar, K. J., \& Taylor, L. S. (2012). Understanding Polymer Properties Important for Crystal Growth Inhibition-Impact of Chemically Diverse Polymers on Solution Crystal Growth of Ritonavir. Crystal Growth \& Design, 12(6), 3133-3143.

Ilevbare, G. A., Liu, H., Edgar, K. J., \& Taylor, L. S. (2013). Impact of Polymers on Crystal Growth Rate of Structurally Diverse Compounds from Aqueous Solution. Molecular Pharmaceutics, 10(6), 2381-2393.

Ilevbare, G. A., \& Taylor, L. S. (2013). Liquid-Liquid Phase Separation in Highly Supersaturated Aqueous Solutions of Poorly Water-Soluble Drugs: Implications for Solubility Enhancing Formulations. Crystal Growth \& Design, 13(4), 1497-1509.

Jaganathan, S. K. (2011). Can flavonoids from honey alter multidrug resistance? Medical Hypotheses, 76(4), 535-537.

Kakran, M., Sahoo, N. G., Li, L., \& Judeh, Z. (2012). Fabrication of quercetin nanoparticles by anti-solvent precipitation method for enhanced dissolution. Powder Technology, 223(0), 59-64.

Konno, H., Handa, T., Alonzo, D. E., \& Taylor, L. S. (2008). Effect of polymer type on the dissolution profile of amorphous solid dispersions containing felodipine. European Journal of Pharmaceutics and Biopharmaceutics, 70(2), 493-499. 
Lauro, M. R., Maggi, L., Conte, U., De Simone, F., \& Aquino, R. P. (2005). Rutin and quercetin gastro-resistant microparticles obtained by spray-drying technique. Journal of Drug Delivery Science and Technology, 15(5), 363-369.

Lauro, M. R., Torre, M. L., Maggi, L., De Simone, F., Conte, U., \& Aquino, R. P. (2002). Fastand Slow-Release Tablets for Oral Administration of Flavonoids: Rutin and Quercetin. Drug Development and Industrial Pharmacy, 28(4), 371-379.

Li, B., Konecke, S., Harich, K., Wegiel, L., Taylor, L. S., \& Edgar, K. J. (2013). Solid dispersion of quercetin in cellulose derivative matrices influences both solubility and stability. Carbohydrate Polymers, 92(2), 2033-2040.

Liu, H., Ilevbare, G. A., Cherniawski, B. P., Ritchie, E. T., Taylor, L. S., \& Edgar, K. J. (2014). Synthesis and structure-property evaluation of cellulose $\omega$-carboxyesters for amorphous solid dispersions. Carbohydrate Polymers, 100, 116-125.

Marks, J. A., Wegiel, L. A., Taylor, L. S., \& Edgar, K. J. (2014). Pairwise Polymer Blends for Oral Drug Delivery. Journal of pharmaceutical sciences, 103(9), 2871-2883.

Pereira, J. M., Mejia-Ariza, R., Ilevbare, G. A., McGettigan, H. E., Sriranganathan, N., Taylor, L. S., Davis, R.M., Edgar, K. J. (2013). Interplay of Degradation, Dissolution and Stabilization of Clarithromycin and Its Amorphous Solid Dispersions. Molecular Pharmaceutics, 10(12), 4640-4653.

Posey-Dowty, J. D., Watterson, T. L., Wilson, A. K., Edgar, K. J., Shelton, M. C., \& Lingerfelt, L. R. (2007). Zero-order release formulations using a novel cellulose ester. Cellulose, 14(1), 73-83.

Price, K. R., Casuscelli, F., Colquhoun, I. J., \& Rhodes, M. J. C. (1998). Composition and content of flavonol glycosides in broccoli florets (Brassica olearacea) and their fate during cooking. Journal of the Science of Food and Agriculture, 77(4), 468-472.

Russo, M., Spagnuolo, C., Tedesco, I., Bilotto, S., \& Russo, G. L. (2012). The flavonoid quercetin in disease prevention and therapy: Facts and fancies. Biochemical Pharmacology, 83(1), 6-15.

Sahu, S., Saraf, S., Kaur, C. D., \& Saraf, S. (2013). Biocompatible Nanoparticles for Sustained Topical Delivery of Anticancer Phytoconstituent Quercetin. Pakistan Journal of Biological Sciences, 16(13), 601-609.

Shelton, M. C., Posey-Dowty, J. D., Lingerfelt, L., Kirk, S. K., Klein, S., \& Edgar, K. J. (2009). Enhanced dissolution of poorly soluble drugs from solid dispersions in carboxymethylcellulose acetate butyrate matrices. In Polysaccharide Materials: Performance by Design (pp. 93-113): American Chemical Society

Song. (2005). Associations of dietary flavonoids with risk of type 2 diabetes, and markers of insulin resistance and systemic inflammation in women: a prospective study and crosssectional analysis. Journal of the American College of Nutrition, 24(5), 376-384.

Srinivas, K., King, J. W., Howard, L. R., \& Monrad, J. K. (2010). Solubility and solution thermodynamic properties of quercetin and quercetin dihydrate in subcritical water. Journal of Food Engineering, 100(2), 208-218.

Tran, T. H., Guo, Y., Song, D., \& Bruno, R. S. Quercetin - Containing Self - Nanoemulsifying Drug Delivery System for Improving Oral Bioavailability. Journal of pharmaceutical sciences, 103(3), 840-852.

Van den Mooter, G., Wuyts, M., Blaton, N., Busson, R., Grobet, P., Augustijns, P., \& Kinget, R. (2001). Physical stabilisation of amorphous ketoconazole in solid dispersions with 
492

493

494

495

496

497

498

499

500

501 polyvinylpyrrolidone K25. European Journal of Pharmaceutical Sciences, 12(3), 261269.

Vasconcelos, T., Sarmento, B., \& Costa, P. (2007). Solid dispersions as strategy to improve oral bioavailability of poor water soluble drugs. Drug Discovery Today, 12(23-24), 10681075.

Zhou, W., Kallifatidis, G., Baumann, B., Rausch, V., Mattern, J., Gladkich, J., Giese, N., Moldenhauer, G, Wirth, T., Buchler, M.W., Salnikov, A.V., Herr, I. (2010). Dietary polyphenol quercetin targets pancreatic cancer stem cells. International journal of oncology, 37(3). 